\title{
PHASE ANGLE AND FRAILTY ARE IMPORTANT PROGNOSTIC FACTORS IN CRITICALLY ILL MEDICAL PATIENTS: A PROSPECTIVE COHORT STUDY
}

\author{
S.J. KO ${ }^{1,2}$, J. CHO ${ }^{1}$, S.M. CHOI ${ }^{1}$, Y.S. PARK ${ }^{1}$, C.-H. LEE ${ }^{1}$, S.-M. LEE ${ }^{1}$, C.-G. YOO ${ }^{1}$, Y.W. KIM ${ }^{1}$, J. LEE ${ }^{1}$ \\ 1. Division of Pulmonary and Critical Care Medicine, Department of Internal Medicine, Seoul National University College of Medicine, Seoul, Republic of Korea; 2. Division of \\ Pulmonary and Critical Care Medicine, Department of Internal Medicine, Wonkwang University Sanbon Hospital, Gunpo, Republic of Korea. Corresponding author: Jinwoo Lee, MD, \\ Department of Internal Medicine, Seoul National University College of Medicine, 103 Daehak-ro, Seoul, 03080, Republic of Korea; E-mail: realrain7@gmail.com
}

\begin{abstract}
Objectives: To investigate whether phase angle (PhA) measured by bioelectrical impedance analysis (BIA) and frailty are associated with the outcomes of critical illnesses. Design: A single-center prospective cohort study. Setting: Medical intensive care unit (ICU) in Seoul National University Hospital, Seoul, Republic of Korea. Participants: 97 patients who were admitted to the medical ICU. Measurements: On admission, PhA was measured by BIA, and frailty was assessed by the Korean Modified Barthel Index (KMBI) scoring system. Patients were classified according to PhA and KMBI scores, and their impact on the outcomes of critical illnesses was evaluated. Results: The patients' mean age was $62.4 \pm 16.4$ years, and 56 of the patients $(57.7 \%)$ were men. Having a high PhA above 3.5 at the time of ICU admission was associated with lower in-hospital mortality (adjusted OR 0.42, $\mathrm{p}=.042$ ), and a shorter duration of ICU stay (5.6 days vs. 9.8 days, $\mathrm{p}=.016$ ) compared to those with a low PhA. Other indices measured by BIA were not significantly associated with outcomes of critical illnesses. Frailty $(\mathrm{KMBI}>60)$ was associated with more mechanical ventilation days (2.3 days vs. 7.1 days; $p$ $=.018)$. Conclusion: Both $\mathrm{PhA}$ and frailty are important prognostic factors predicting the outcomes of critical illnesses. Low PhA scores were associated with increased mortality and a longer duration of ICU stay, and frailty was associated with more mechanical ventilation days.
\end{abstract}

Key words: Bioelectrical impedance analysis, phase angle, frailty.

\section{Introduction}

Patients admitted to the intensive care unit (ICU) usually have many underlying diseases and need critical care for various reasons. Given the heterogeneity of ICU patients, outcome prediction is difficult. Although severity scoring systems combining multiple variables including vital signs, anthropometric and laboratory data such as Acute Physiology And Chronic Health Evaluation (APACHE) and Sequential Organ Failure Assessment (SOFA) have been introduced, their interpretation of the course and outcomes of critical illnesses are limited (1-5). Many attempts have been made to find new, relevant prognostic factors in critically ill patients.

Bioelectrical impedance analysis (BIA) is a method of measuring body compartments using the electrical properties of tissues (6). It is a non-invasive test and does not require special training to use. BIA is frequently used in the general population to assess body composition, in particular muscle mass and fat. Recently, its results have been comparable to that of dual X-ray absortiometry, the gold standard method for assessing body composition, including muscle mass, fat mass and body cell mass (7). Unlike dual X-ray absorptiometry, however, BIA can be easily applied to patients in the ICU.

Phase angle ( $\mathrm{PhA})$ as measured by BIA is known to reflect cell membrane integrity, permeability and fat-free mass, and higher values of PhA suggest better cell health, gains in cell function, and better nutritional status (8). With its reliability and generalizability to healthy populations (9), $\mathrm{PhA}$ is being investigated as a useful index in various diseases and has been proven to be an independent prognostic factor for several kind of cancers, including advanced lung and pancreatic, head and neck cancer (10-12), and breast cancer (13). A few studies have investigated the usefulness of $\mathrm{PhA}$ in critically ill patients and have revealed that low values of $\mathrm{PhA}$ were associated with higher mortality of patients in the ICU $(8,14-20)$ or longer ICU stay $(18,21)$. However, studies on critically ill medical patients in Asia are very rare $(16,19,20)$.

Although more elderly patients are being admitted to the ICU, age alone is an unreliable factor in predicting the prognosis of critical illnesses (22-24). Frailty is defined as a multi-dimensional syndrome characterized by the loss of physical and cognitive reserves, leading to increased vulnerability to possible stressors (25). Thus, frailty, as measured by various frailty scoring systems, is considered to be an important factor influencing the outcomes of critical illnesses $(26,27)$. Recent studies have revealed that frailty is associated with higher mortality $(28,29)$, extubation failure, and prolonged ventilation period (30) in critically ill patients.

The aim of this study was to assess the value of PhA and frailty on predicting the outcome of critically ill medical patients.

\section{Methods}

\section{Study design and participants}

This was a prospective cohort study of patients admitted to the medical ICU (MICU) of a university-affiliated teaching hospital. All patients aged over 19 years and admitted to the 
THE JOURNAL OF NUTRITION, HEALTH \& AGING

Table 1

Baseline characteristics of the patients

\begin{tabular}{|c|c|c|c|c|}
\hline & All patients $(n=97)$ & Survivors $(n=57)$ & Non-survivors $(n=40)$ & p value \\
\hline Age (year) & $62.4 \pm 16.4$ & $63.6 \pm 16.8$ & $60.7 \pm 15.9$ & 0.395 \\
\hline Male sex $(n, \%)$ & $56(57.7 \%)$ & $34(59.6 \%)$ & $22(55.0 \%)$ & 0.648 \\
\hline Body weight (kg) & $61.7 \pm 13.6$ & $62.9 \pm 15.3$ & $60.0 \pm 10.7$ & 0.318 \\
\hline Height $(\mathrm{cm})$ & $163.4 \pm 8.9$ & $164.8 \pm 10.0$ & $161.4 \pm 6.6$ & 0.065 \\
\hline APACHE II score & $19.0 \pm 7.6$ & $18.4 \pm 8.4$ & $20.0 \pm 6.3$ & 0.319 \\
\hline SOFA score & $8.0 \pm 3.9$ & $7.4 \pm 3.3$ & $8.8 \pm 4.6$ & 0.095 \\
\hline Reasons for ICU admission (n)* & 128 & 75 & 53 & \\
\hline Respiratory failure (n, \%) & $61(62.9 \%)$ & $37(49.3 \%)$ & $24(45.3 \%)$ & 0.651 \\
\hline Renal failure (n, \%) & $27(27.8 \%)$ & $12(16.0 \%)$ & $15(28.3 \%)$ & 0.161 \\
\hline Cardiovascular cause $(\mathrm{n}, \%)$ & $17(17.5 \%)$ & $12(16.0 \%)$ & $5(9.4 \%)$ & 0.281 \\
\hline Sepsis $(\mathrm{n}, \%)$ & $14(14.4 \%)$ & $7(9.3 \%)$ & $7(13.2 \%)$ & 0.489 \\
\hline Neurologic cause (n, \%) & $2(2.1 \%)$ & $1(1.3 \%)$ & $1(1.9 \%)$ & 0.804 \\
\hline Others $(\mathrm{n}, \%)$ & $7(7.2 \%)$ & $6(8.0 \%)$ & $1(1.9 \%)$ & 0.134 \\
\hline \multicolumn{5}{|l|}{ BIA values } \\
\hline Body mass index $\left(\mathrm{kg} / \mathrm{m}^{2}\right)$ & $23.1 \pm 4.0$ & $23.0 \pm 4.3$ & $23.1 \pm 3.5$ & 0.872 \\
\hline Muscle mass (kg) & $44.8 \pm 10.3$ & $46.0 \pm 10.8$ & $43.2 \pm 9.3$ & 0.200 \\
\hline Fat-free mass (kg) & $47.6 \pm 10.9$ & $48.7 \pm 11.4$ & $46.1 \pm 9.9$ & 0.247 \\
\hline Body fat $(\%)$ & $22.8 \pm 11.5$ & $22.0 \pm 11.1$ & $28.4 \pm 12.0$ & 0.439 \\
\hline PhA score & $3.6 \pm 1.2$ & $3.8 \pm 1.2$ & $3.3 \pm 1.3$ & 0.077 \\
\hline Proportion of $\mathrm{PhA} \geq 3.5(\%)$ & $46(47.4 \%)$ & $32(56.1 \%)$ & $14(35.0 \%)$ & 0.040 \\
\hline KMBI score & $63.7 \pm 40.7$ & $62.5 \pm 41.8$ & $65.5 \pm 39.5$ & 0.727 \\
\hline Proportion of KMBI > $60(\%)$ & $57(58.8 \%)$ & $34(59.6 \%)$ & $23(57.5 \%)$ & 0.832 \\
\hline
\end{tabular}

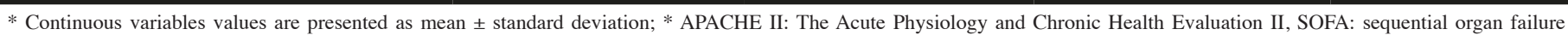

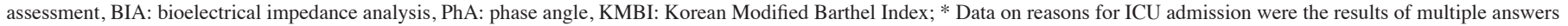

MICU were screened for eligibility from June 2017 to May 2018. Patients who were admitted for planned procedures only (coronary angiography, bronchoscopy, tracheostomy, etc.), or for short-term close observation after planned surgery, were not included.

This study was approved by the institutional review board of Seoul National University Hospital (H-1704-140-848). All patients provided written informed consent and the study was conducted in accordance with the tenets of the Declaration of Helsinki.

\section{Data collection}

After enrollment, anthropometric characteristics including age, sex, body weight, height were recorded. The reasons for admission to ICU were also recorded. Severity indices including APACHE II and sequential organ failure assessment (SOFA) scores were calculated. Patients' current general health status was evaluated by various methods. To evaluate frailty, we used the Modified Barthel Index, which was revised and validated as the Korean Modified Barthel Index (KMBI) (31).

BIA was measured using a portable BIA device (InBody $\mathrm{S} 10^{\circ, \mathrm{R}}$, InBody Corp., Seoul, South Korea) within $24 \mathrm{~h}$ after enrollment. The InBody S10 ${ }^{\circ} \mathrm{R}$ was designed to be applicable to patients in the supine position, so almost all critically ill patients could be analyzed. Patient information including age, sex, body weight, and height were entered into the BIA device, and electrodes were attached on each thumb, third finger, and ankle. After electrical currents of 1, 5, 50, 250, 500, and $1,000 \mathrm{MHz}$ were applied through the electrodes, whole body intracellular water, extracellular water, protein, mineral, body fat mass, and skeletal muscle mass were calculated. Whole body resistance $(\mathrm{R})$ and reactance $(\mathrm{Xc})$ were measured upon the electrical current of $50 \mathrm{MHz}$, and then the phase angle was calculated by the following formula: $\mathrm{PhA}\left({ }^{\circ}\right)=\arctan (\mathrm{Xc} / \mathrm{R}) \times$ $(180 / \pi)(13)$.

All enrolled patients were followed until they were discharged. Data on survival status when they were discharged from the ICU or hospital, the length of stay in the ICU, and the 
number of mechanically ventilated days were recorded.

\section{Statistical analysis}

The patient's age, sex, body mass index (BMI), APACHE II score, SOFA score, $\mathrm{PhA}$, and KMBI score were analyzed as independent variables to determine whether they were associated with in-hospital mortality. For the categorical analysis, we divided the participants into two groups according to $\mathrm{PhA}$ and KMBI. The cutoff to divide the two groups was selected, based on the results from previous studies $(8,32)$.

To determine the independent effect of $\mathrm{PhA}$ and KMBI values on the outcomes of critical illnesses, we conducted a multiple regression analysis by adjusting for age, sex, BMI, APACHE II score, and SOFA score. We evaluated whether the duration of ICU care differed according to PhA values through a Kaplan-Meier curve analysis. Additionally, we evaluated whether the number of mechanically ventilated days differed according to the KMBI scores. To determine the relationship between BIA values and KMBI, we used Pearson correlation analysis.

Statistical analyses were performed using SPSS software (version 25.0 for Windows; IBM SPSS Inc., Armonk, NY, USA) and R (version 4.0.0, https://www.R-project.org/). All statistical tests were two-sided, and differences were considered statistically significant at $\mathrm{p}<0.05$.

\section{Results}

\section{Study population}

Among the 115 patients enrolled in the cohort, 97 patients who underwent KMBI and BIA measurements at the time of admission were included for analysis. Baseline characteristics of the patients including anthropometric data, hospital route and reasons for ICU admission, disease severity scores, $\mathrm{PhA}$, and KMBI are shown in Table 1.

During the ICU stay, 51 patients (52.6\%) were mechanically ventilated, and 33 patients $(34.0 \%)$ received renal replacement therapy. Eighteen patients (18.6\%) were treated for sepsis. The ICU mortality of the study population was $25.8 \%$. The mean duration of the ICU stay among the survivors was $7.7 \pm 7.5$ days, and the mean number of mechanically ventilated days among intubated ICU survivors was $4.8 \pm 6.3$ days.

\section{Factors associated with all-cause in-hospital mortality}

The all-cause in-hospital mortality was $41.2 \%$. After multiple logistic regression, adjusting for age, sex, BMI, APACHE II score, SOFA score, and KMBI, the group with higher $\mathrm{PhA}$ values $(\mathrm{PhA} \geq 3.5)$ was independently associated with decreased in-hospital mortality compared to the group with lower $\mathrm{PhA}$ values (odds ratio 0.42, $\mathrm{p}=.042$ ). The other variables, including APACHE II, SOFA score, and KMBI were not associated with in-hospital mortality (Table 2).

Other variables from the BIA measurement including intracellular water(ICW), extracellular water(ECW), total body
water(TBW), ECW/TBW, ECW/body weight, protein, mineral, body fat mass, body fat rate, visceral fat area, muscle mass, fat-free mass(FFM), TBW/FFM were entered into the multiple logistic regression analysis, but none were independently associated with in-hospital mortality (Table 2).

Table 2

Independent predictors of in-hospital mortality by multiple logistic regression analysis

\begin{tabular}{lcc}
\hline Independent variables & $\begin{array}{c}\text { Odds ratio } \\
\text { (95\% confidence interval) }\end{array}$ & p value \\
\hline Age & $0.99(0.96-1.01)$ & 0.391 \\
Sex & $1.21(0.53-2.75)$ & 0.648 \\
APACHE II score & $1.03(0.97-1.09)$ & 0.318 \\
SOFA score & $1.09(0.99-1.22)$ & 0.098 \\
BIA values & & \\
Body mass index & $1.01(0.91-1.12)$ & 0.871 \\
ICW & $0.94(0.86-1.02)$ & 0.152 \\
ECW & $0.95(0.84-1.06)$ & 0.365 \\
TBW & $0.97(0.92-1.02)$ & 0.214 \\
ECW/TBW & $1.11(0.94-1.37)$ & 0.287 \\
ECW/body weight & $0.98(0.88-1.09)$ & 0.705 \\
Protein & $0.86(0.69-1.05)$ & 0.137 \\
Mineral & $1.14(0.72-1.83)$ & 0.572 \\
Body fat mass & $1.01(0.96-1.05)$ & 0.825 \\
Body fat rate & $1.01(0.98-1.05)$ & 0.435 \\
Visceral fat area & $1.00(0.99-1.01)$ & 0.488 \\
Muscle mass & $0.97(0.93-1.01)$ & 0.200 \\
FFM & $0.98(0.94-1.02)$ & 0.245 \\
TBW/FFM & $0.72(0.43-1.13)$ & 0.176 \\
PhA $\geq 3.5$ & $0.42(0.18-0.96)$ & 0.042 \\
KMBI > 60 & $0.92(0.40-2.09)$ & 0.832 \\
\hline * APACHE II: The Acute Physiology and Chronic Health Evaluation II, SOFA: \\
sequential organ failure assessment, BIA: bioelectrical impedance analysis, ICW: \\
intracellular water, ECW: extracellular water, TBW: total body water, FFM: fat-free \\
mass, PhA: phase angle, KMBI: Korean Modified Barthel Index & \\
& &
\end{tabular}

The mean KMBI score of the survivors was not significantly different from that of the nonsurvivors (62.5 vs. $65.5, \mathrm{p}=.727)$.

Factors associated with duration of ICU stay in ICU survivors

We analyzed the association between $\mathrm{PhA}$ values and the duration of the ICU stay among the 72 ICU survivors. The patients with higher $\mathrm{PhA}$ values recovered and were discharged from the ICU earlier compared to those with lower PhA values (ICU days 5.6 vs. 9.8, $\mathrm{p}=.016$ ). The Kaplan-Meier analysis showed the superiority of higher $\mathrm{PhA}$ values compared with lower PhA values $(\mathrm{p}=.011)$ (Figure 1$)$. Higher SOFA score 
was also associated with longer duration of ICU stay $(\mathrm{p}=.004)$. The KMBI score was not associated with duration of ICU stay $(\mathrm{p}=.232)$.

Figure 1

Kaplan-Meier plot of intensive care unit discharge according to $\mathrm{PhA}$ group

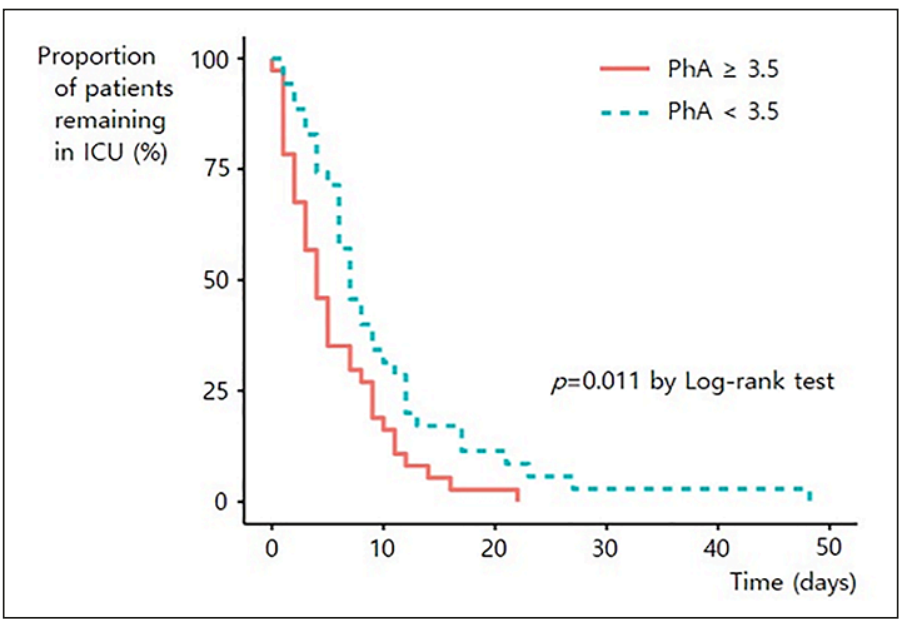

Factors associated with mechanically ventilated days in ICU survivors

Among 72 ICU survivors, 32 patients who were intubated were included in this analysis. The Kaplan-Meier analysis showed that less frail patients, that is, those with a higher KMBI score $(\mathrm{KMBI}>60)$ were weaned from mechanical ventilation earlier compared to more frail patients (2.3 days vs. 7.1 days; $\mathrm{p}=.018$ ) (Figure 2). Multiple regression analysis revealed that none of the BIA values, including $\mathrm{PhA}$, were associated with the number of mechanically ventilated days (Table 3).

Figure 2

Kaplan-Meier plot of ventilator weaning according to KMBI group

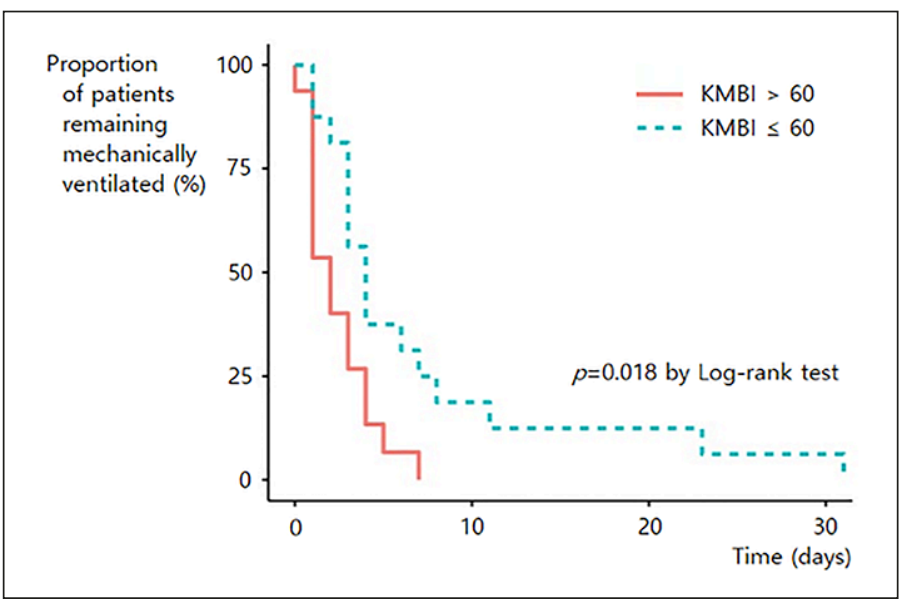

Table 3

Association between BIA values and mechanically ventilated days by multiple regression analysis

\begin{tabular}{lcc}
\hline BIA values & Beta-coefficient & p value \\
\hline ICW & 0.12 & 0.519 \\
ECW & 0.18 & 0.328 \\
TBW & 0.14 & 0.434 \\
ECW/TBW & 0.14 & 0.456 \\
ECW/body weight & 0.14 & 0.449 \\
Protein & 0.13 & 0.494 \\
Mineral & 0.11 & 0.546 \\
Body fat mass & -0.10 & 0.568 \\
Body fat rate & -0.12 & 0.525 \\
Visceral fat area & -0.05 & 0.768 \\
Muscle mass & 0.14 & 0.444 \\
FFM & 0.14 & 0.451 \\
TBW/FFM & 0.18 & 0.324 \\
PhA $\geq 3.5$ & -0.02 & 0.898 \\
\hline
\end{tabular}

* BIA: bioelectrical impedance analysis, ICW: intracellular water, ECW: extracellular water, TBW: total body water, FFM: fat-free mass, PhA: phase angle

Table 4

Correlation analysis between KMBI and BIA values

\begin{tabular}{lcc}
\hline BIA values & r value & p value \\
\hline ICW & 0.24 & 0.016 \\
ECW & 0.12 & 0.258 \\
TBW & 0.19 & 0.057 \\
ECW/TBW & -0.27 & 0.009 \\
ECW/body weight & 0.08 & 0.415 \\
Protein & 0.24 & 0.019 \\
Mineral & 0.23 & 0.026 \\
Body fat mass & -0.18 & 0.072 \\
Body fat rate & -0.22 & 0.029 \\
Visceral fat area & -0.28 & 0.005 \\
Muscle mass & 0.21 & 0.044 \\
FFM & 0.21 & 0.040 \\
TBW/FFM & -0.22 & 0.031 \\
PhA & 0.40 & $<0.001$ \\
\hline
\end{tabular}

* KMBI: Korean Modified Barthel Index, BIA: bioelectrical impedance analysis, ICW: intracellular water, ECW: extracellular water, TBW: total body water, FFM: fat-free mass, $\mathrm{PhA}$ : phase angle

\section{Correlation between frailty and BIA values}

KMBI was positively or negatively correlated with several BIA values. Phase angle was most strongly correlated with KMBI among BIA values $(\mathrm{r}=0.40, \mathrm{p}<.001)$. ICW, protein, 


\section{PHASE ANGLE AND FRAILTY IN ICU}

mineral, muscle mass and FFM were positively correlated with $\mathrm{KMBI}$, and ECW/TBW, body fat rate, visceral fat area, TBW/ FFM were negatively correlated with KMBI (Table 4).

\section{Discussion}

Our study revealed that low $\mathrm{PhA}$ values, as measured by BIA, were independently associated with increased all-cause in-hospital mortality in the patients admitted to a MICU. Additionally, $\mathrm{PhA}$ and frailty were both important indices related to the prognosis of critically ill patients, each of which provided different information to clinicians.

The use of BIA is becoming more popular in the medical field, presenting significant usefulness in evaluating nutritional and muscle status and also in predicting the prognosis of chronic diseases and cancers. However, only recently has BIA been used in the field of critical care. Among the many values derived using the technique of $\mathrm{BIA}, \mathrm{PhA}$ is an interesting value, and it represents cell health and functional status as estimated by the indices of body composition. By virtue of its usefulness in assessing overall health status, $\mathrm{PhA}$ measurements through BIA in ICU patients may also help clinicians assess and predict the prognosis of critically ill patients.

Previous studies on PhA and mortality were mostly not focused on the critical ill medical patients $(8,14,15)$. Only three studies from Asia are available, but they only included critically ill surgical patients (20) or retrospectively gathered a small number of patients with low mortality (16). More than $1 / 3$ of the study population of another study were patients from acute pancreatitis, which limits the generalizability of the results (19). Moreover, lower PhA was associated with prolonged duration of ICU stay as well as all-cause in-hospital mortality of critically ill patients. This is comparable to other studies suggesting the association of $\mathrm{PhA}$ and duration of ICU stay $(18,21)$.

The mean PhA value of our study population was 3.6, which is much lower than that of the normal population showing the mean PhA above 5.0 (9). The value of PhA in critically ill patients is usually low (16-19), indicating severely injured cell membrane integrity and increased cell membrane permeability due to critical illness. Although the optimal cutoff of PhA value associated with increased mortality is not determined, a large study suggested using a cutoff of PhA 3.49 (8), which is consistent with our study. A recent study used a cutoff of 5.5 and were not able to show differences in mortality (21). A lower PhA cutoff of 3.8 revealed increased mortality with lower $\mathrm{PhA}$ in critically ill cancer patients (18). These results suggest that it may be reasonable to use a cutoff of PhA 3.49-3.8 to predict the outcomes of critical illnesses.

This study also highlights the importance of frailty in the context of critical care. Due to the improvements in the health care system and increased interest in the management of chronic diseases, aging itself is not an independent prognostic factor of critical illnesses anymore (22-24). Frailty, however, leads to vulnerability to adverse outcomes of various diseases, including critical illnesses $(28,29)$. KMBI used in this study to assess a patient's frailty, evaluates personal hygiene and bathing, feeding, dressing, stair climbing, bowel and bladder control, ambulation, and stair climbing. It enables a multidimensional and extensive approach to assess patients' functional status in patients with critical illnesses (33).Weaning from mechanical ventilation requires the cooperative function of multiple organ systems including respiratory system, musculoskeletal system, and mental effort (34). Frail patients with disorganized functioning are challenged when intubated, and they often find it difficult to spontaneously breathe without the support of the ventilator. Frail patients in our study population also had difficulty weaning from the ventilator which led to more mechanical ventilation days.

An association between frailty and $\mathrm{PhA}$ values have been suggested in the general population and in patients with specific diseases (35-38), but it has not been studied in the context of critical illness outcomes. In this study, PhA and KMBI measured at the admission to ICU showed positive correlation. KMBI was also correlated positively with BIA values indicating better health status such as FFM, and negatively with those indicating poor health status such as ECW/TBW, body fat rate, and visceral fat area. It is interesting to note that even in critically ill patients, low PhA which represents poor cell health is associated with frailty which represents general wellbeing of a patient.

Our study has several limitations. First, conventional severity scoring systems such as APACHE II and SOFA were not able to predict the survival of our study population. Discrimination by a scoring system can change over time, due to changing patterns and severity of illnesses at different time points (1), and due to discrepancies between various scoring systems according to targeted populations or diseases $(2,3,5)$. Second, this was a single-center study that included patients with a relatively high number of comorbid conditions with high severity. This situation reflected the characteristics of a university-affiliated teaching hospital, and it could influence the generalizability of our results.

Despite these limitations, our study presents several strengths. To the best of our knowledge, this is the first study to investigate the influence of both the $\mathrm{PhA}$ and frailty on the outcomes of critical illnesses in a well-defined medical ICU population. Our study participants represent the critical stage of various medical illnesses. We enrolled participants prospectively, which excluded selection bias and provided highquality data.

In conclusion, low $\mathrm{PhA}$ values were independently associated with increased all-cause in-hospital mortality in the patients with critical illnesses in the MICU. PhA and frailty were important prognostic factors predicting the outcomes of critical illnesses, especially mortality and mechanical ventilation weaning failure, respectively. Assessing $\mathrm{PhA}$ and frailty of critically ill patients at ICU admission might help 


\section{THE JOURNAL OF NUTRITION, HEALTH \& AGING}

predict the outcomes of critical illnesses in the ICU. Further studies are necessary to investigate the mechanism of the association between $\mathrm{PhA}$, frailty and the course of critical illnesses.

Conflict of interest: None declared.

Funding/Support: No funding was received for this study.

Notation of prior abstract publication/presentation: None declared.

Ethical standards: The current research was approved by the institutional review board of Seoul National University Hospital and the approval number is H-1704-140-848.

\section{References}

1. Varghese YE, Kalaiselvan MS, Renuka MK, Arunkumar AS. Comparison of acute physiology and chronic health evaluation II (APACHE II) and acute physiology and chronic health evaluation IV (APACHE IV) severity of illness scoring systems, in a multidisciplinary ICU. J Anaesthesiol Clin Pharmacol 2017;33(2):248-253.

2. Park S-K, Chun H-J, Kim D-W, Im T-H, Hong H-J, Yi H-J. Acute Physiology an Chronic Health Evaluation II and Simplified Acute Physiology Score II in predicting hospital mortality of neurosurgical intensive care unit patients. J Korean Med Sci 2009;24(3):420-426.

3. Raj R, Skrifvars MB, Bendel S, Selander T, Kivisaari R, Siironen J, et al. Predicting six-month mortality of patients with traumatic brain injury: usefulness of common intensive care severity scores. Crit care 2014;18(2):R60.

4. Schoe A, Bakhshi-Raiez F, de Keizer N, van Dissel JT, de Jonge E. Mortality prediction by SOFA score in ICU-patients after cardiac surgery; comparison with traditional prognostic-models. BMC Anesthesiol 2020;20(1):1-8.

5. Ko M, Shim M, Lee S-M, Kim Y, Yoon S. Performance of APACHE IV in medical intensive care unit patients: Comparisons with APACHE II, SAPS 3, and MPMO III. Acute Crit Care 2018;33(4):216-221

6. Kyle UG, Bosaeus I, De Lorenzo AD, Deurenberg P, Elia M, Gómez JM, et al. Bioelectrical impedance analysis - part I: review of principles and methods. Clin Nutr 2004;23(5): 1226-1243.

7. Kafri MW, Potter JF, Myint PK. Multi-frequency bioelectrical impedance analysis for assessing fat mass and fat-free mass in stroke or transient ischaemic attack patients. Eur J Clin Nutr 2014;68(6):677-682.

8. Thibault R, Makhlouf A-M, Mulliez A, Gonzalez MC, Kekstas G, Kozjek NR, et al Fat-free mass at admission predicts 28-day mortality in intensive care unit patients: the international prospective observational study Phase Angle Project. Intensive Care Med 2016;42(9):1445-1453.

9. Bosy-Westphal A, Danielzik S, Dörhöfer R, Later W, Wiese S, Müller MJ. Phase angle from bioelectrical impedance analysis: population reference values by age, sex, and body mass index. J Parenter Enter Nutr 2006;30(4):309-316.

10. Gupta D, Lammersfeld CA, Vashi PG, King J, Dahlk SL, Grutsch JF, et al. Bioelectrical impedance phase angle in clinical practice: implications for prognosis in stage IIIB and IV non-small cell lung cancer. BMC Cancer 2009;9(1):37.

11. Gupta D, Lis CG, Dahlk SL, Vashi PG, Grutsch JF, Lammersfeld CA. Bioelectrical impedance phase angle as a prognostic indicator in advanced pancreatic cancer. Br J Nutr 2004;92(6):957-962.

12. Axelsson L, Silander E, Bosaeus I, Hammerlid E. Bioelectrical phase angle at diagnosis as a prognostic factor for survival in advanced head and neck cancer. Eur Arch Oto-Rhino-Laryngology 2018;275(9):2379-2386

13. Gupta D, Lammersfeld CA, Vashi PG, King J, Dahlk SL, Grutsch JF, et al. Bioelectrical impedance phase angle as a prognostic indicator in breast cancer. BMC Cancer 2008;8(1):249.

14. Buter H, Veenstra JA, Koopmans M, Boerma CE. Phase angle is related to outcome after ICU admission; an observational study. Clin Nutr ESPEN 2018;23:61-66.

15. Stapel SN, Looijaard WGPM, Dekker IM, Girbes ARJ, Weijs PJM, Oudemans-van Straaten HM. Bioelectrical impedance analysis-derived phase angle at admission as a predictor of 90-day mortality in intensive care patients. Eur J Clin Nutr 2018;72(7):1019-1025.
16. Lee Y, Kwon O, Shin CS, Lee SM. Use of bioelectrical impedance analysis for the assessment of nutritional status in critically ill patients. Clin Nutr Res 2015;4(1):32-40.

17. Ellegård LH, Petersen P, Öhrn L, Bosaeus I. Longitudinal changes in phase angle by bioimpedance in intensive care patients differ between survivors and non-survivors Clin Nutr ESPEN 2018;24:170-172.

18. do Amaral Paes TC, de Oliveira KCC, de Carvalho Padilha P, Peres WAF. Phase angle assessment in critically ill cancer patients: relationship with the nutritional status, prognostic factors and death. J Crit Care 2018;44:430-435.

19. Jiashu Y, Minlin Z, Bin X, Chuan L, Haiyan C, Dehua G. The association of bioimpedance analysis parameters with the outcomes of critically ill patients. Clin Nutr 2019 Dec 23. Online ahead of print.

20. Lee YH, Lee J-D, Kang DR, Hong J, Lee J. Bioelectrical impedance analysis values as markers to predict severity in critically ill patients. J Crit Care 2017;40:103-107.

21. Razzera EL, Marcadenti A, Rovedder SW, Alves FD, Fink J da S, Silva FM. Parameters of bioelectrical impedance are good predictors of nutrition risk, length of stay, and mortality in critically Ill patients: a prospective cohort study. J Parenter Enter Nutr 2019 Aug 18. Online ahead of print.

22. McDermid RC, Stelfox HT, Bagshaw SM. Frailty in the critically ill: a novel concept. Crit Care 2011:15(1):301.

23. Lee SH, Lee TW, Ju S, Yoo J-W, Lee SJ, Cho YJ, et al. Outcomes of very elderly ( $\geq$ 80 years) critical-ill patients in a medical intensive care unit of a tertiary hospital in Korea. Korean J Intern Med 2017;32(4):675-681.

24. Yoo J-W, Ju S, Lee SJ, Cho M-C, Cho YJ, Jeong YY, et al. Characteristics an Outcomes of Patients with Pulmonary Acute Respiratory Distress Syndrome Infected with Influenza versus Other Respiratory Viruses. Tuberc Respir Dis (Seoul) 2019;82(4):328-334.

25. Jung H-W, Yoo H-J, Park S-Y, Kim S-W, Choi J-Y, Yoon S-J, et al. The Korean version of the FRAIL scale: clinical feasibility and validity of assessing the frailty status of Korean elderly. Korean J Intern Med 2016;31(3):594-600.

26. Singer JP, Lederer DJ, Baldwin MR. Frailty in pulmonary and critical care medicine. Ann Am Thorac Soc 2016;13(8):1394-1404

27. Mahalingam M, Moore JX, Donnelly JP, Safford MM, Wang HE. Frailty syndrome and risk of sepsis in the REasons for Geographic and Racial Differences in Stroke (REGARDS) Cohort. J Intensive Care Med 2019;34(4):292-300.

28. Brummel NE, Bell SP, Girard TD, Pandharipande PP, Jackson JC, Morandi A, et al. Frailty and subsequent disability and mortality among patients with critical illness. Am J Respir Crit Care Med 2017;196(1):64-72.

29. Flaatten H, De Lange DW, Morandi A, Andersen FH, Artigas A, Bertolini G, et al The impact of frailty on ICU and 30-day mortality and the level of care in very elderly patients ( $\geq 80$ years). Intensive Care Med 2017;43(12):1820-1828.

30. Fernando SM, McIsaac DI, Rochwerg B, Bagshaw SM, Muscedere J, Munshi L, et al. Frailty and invasive mechanical ventilation: association with outcomes, extubation failure, and tracheostomy. Intensive Care Med 2019;45(12):1742-1752.

31. Jung HY, Park BK, Shin HS, Kang YK, Pyun SB, Paik NJ, et al. Development of the Korean Version of Modified Barthel Index (K-MBI): Multi-center Study for Subjects with Stroke. J Korean Acad Rehabil Med 2007;31(3):283-297.

32. Shah S, Vanclay F, Cooper B. Improving the sensitivity of the Barthel Index for stroke rehabilitation. J Clin Epidemiol 1989;42(8):703-709.

33. Villa P, Pintado M, Luján J, González-García N, Trascasa M, Molina R, et al Functional status and quality of life in elderly intensive care unit survivors. J Am Geriatr Soc 2016;64(3):536-542.

34. McConville JF, Kress JP. Weaning patients from the ventilator. N Engl J Med 2012;367(23):2233-2239.

35. Wilhelm-Leen ER, Hall YN, Horwitz RI, Chertow GM. Phase angle, frailty and mortality in older adults. J Gen Intern Med 2014;29(1):147-154.

36. Delgado C, Doyle JW, Johansen KL. Association of frailty with body composition among patients on hemodialysis. J Ren Nutr 2013;23(5):356-362.

37. Pinheiro M, Mancio J, Conceição G, Ferreira W, Carvalho M, Santos A, et al. Frailty syndrome: Visceral adipose tissue and frailty in patients with symptomatic severe aortic stenosis. J Nutr Health Aging 2017;21(1):120-128.

38. Mullie L, Obrand A, Bendayan M, Trnkus A, Ouimet M, Moss E, et al. Phase angle as a biomarker for frailty and postoperative mortality: the BICS study. J Am Heart Assoc 2018;7(17):e008721. 